\title{
Consensus communication on early peanut introduction and the prevention of peanut allergy in high-risk infants
}

\author{
David M. Fleischer ${ }^{1 \dagger}$, Scott Sicherer ${ }^{2 \dagger}$, Matthew Greenhawt ${ }^{3 \dagger}$, Dianne Campbell ${ }^{4 \dagger}$, Edmond S. Chan ${ }^{5^{* \dagger}}$, \\ Antonella Muraro ${ }^{6 \dagger}$, Susanne Halken ${ }^{6 \dagger}$, Yitzhak Katz ${ }^{7 \dagger}$, Motohiro Ebisawa ${ }^{8 \dagger}$, Lawrence Eichenfield ${ }^{9 \dagger}$, \\ Hugh Sampson ${ }^{10 \dagger}$, FOR THE LEAP TRIAL TEAM and SECONDARY CONTRIBUTORS
}

\begin{abstract}
The purpose of this brief communication is to highlight emerging evidence to existing guidelines regarding potential benefits of supporting early, rather than delayed, peanut introduction during the period of complementary food ntroduction in infants. This document should be considered as interim guidance based on consensus among the following organizations: American Academy of Allergy, Asthma \& Immunology; American Academy of Pediatrics; American College of Allergy, Asthma \& Immunology; Australasian Society of Clinical Immunology and Allergy; Canadian Society of Allergy and Clinical Immunology; European Academy of Allergy and Clinical Immunology; Israel Association of Allergy and Clinical Immunology; Japanese Society for Allergology; Society for Pediatric Dermatology; and World Allergy Organization. More formal guidelines regarding early-life, complementary feeding practices and the risk of allergy development will follow in the next year from the National Institute of Allergy and Infectious Diseases - sponsored Working Group and the European Academy of Allergy and Clinical Immunology.
\end{abstract}

Keywords: Allergy prevention, Complementary feeding, Peanut allergy

\section{Introduction and rationale}

Peanut allergy is an increasingly troubling global health problem affecting between $1 \%$ and $3 \%$ of children in many westernized countries. Although multiple methods of measurement have been used and specific estimates differ, there appears to be a sudden increase in the number of cases in the past 10- to 15-year period, suggesting that the prevalence might have tripled in some countries, such as the United States. Extrapolating the currently estimated prevalence, this translates to nearly 100,000 new cases annually (in the United States and United Kingdom), affecting some 1 in 50 primary school-aged children in the United States, Canada, the United Kingdom, and Australia. A similar rise in incidence is now being noted in developing countries, such as Ghana [1-6].

\footnotetext{
* Correspondence: echan5@cw.bc.ca

${ }^{\dagger}$ Each contributed equally to this work as Primary Contributors

${ }^{5}$ Canadian Society of Allergy and Clinical Immunology (CSACI), Vancouver BC, Canada

Full list of author information is available at the end of the article
}

The purpose of this brief communication is to highlight emerging evidence for existing allergy prevention guidelines regarding potential benefits of supporting early rather than delayed peanut introduction during the period of complementary food introduction in infants. A recent study, entitled "Randomized trial of peanut consumption in infants at risk for peanut allergy" demonstrated a successful $11 \%$ to $25 \%$ absolute reduction in the risk of peanut allergy in high-risk infants (and a relative risk reduction of up to $80 \%$ ) if peanut was introduced between 4 and 11 months of age [7]. In light of the significance of these findings, this document serves to better inform the decision-making process for healthcare providers regarding such potential benefits of early peanut introduction. More formal guidelines regarding early-life, complementary feeding practices and the risk of allergy development will follow in the next year from the National Institute of Allergy and Infectious Diseases (NIAID)-sponsored Working Group and the European Academy of Allergy and Clinical Immunology (EAACI), 
and thus this document should be considered as interim guidance.

\section{Summary of new evidence}

In the Learning Early About Peanut Allergy (LEAP) trial, 640 high-risk United Kingdom infants (See Box 1) between the ages of 4 to 11 months were randomized to consume peanut products at least three times a week ( $6 \mathrm{~g}$ of peanut protein; equivalent to $24 \mathrm{~g}$ peanuts or 3 teaspoons of peanut butter per week) or to completely avoid peanut products for the first 5 years of life. This included 542 infants found to have negative skin prick test (SPT) responses to peanut at study entry, and 98 infants with SPT wheal diameters to peanut of between 1 and $4 \mathrm{~mm}$ (minimally positive SPT response) at study entry. An additional 76 children were excluded from study entry before randomization based on an SPT response of greater than $5 \mathrm{~mm}$, which was assumed to result in a very high likelihood of reacting to a peanut challenge. In an intention-to-treat analysis, $17.2 \%$ in the peanut avoidance group compared to $3.2 \%$ in the peanut consumption group had food challenge-proved peanut allergy by age 5 years, corresponding to a $14 \%$ absolute risk reduction, a number needed to treat (NNT, eg, number of persons needed to be treated for one to receive benefit) of 7.1, and a relative risk reduction of $81 \%$ [7].

When examined in further detail, the isolated beneficial effects for both the primary and secondary prevention of peanut allergy translated to an NNT of 8.5 among the infants with negative SPT responses and an NNT of 4 among the infants with minimally positive SPT responses. Secondary analyses also showed similar levels of prevention in white, black and Asian (Indian and Pakistani) children. Overall, the risk of early introduction in this group was low: 7 of the 319 children randomized to the consumption group reacted to peanut at the baseline food challenge, suggesting that peanut food challenges and introduction, even in infants with minimally positive SPT responses, are safe and feasible. Six children in the consumption group had peanut allergy during the study, indicating that peanut allergy can still develop despite attempts at primary and secondary prevention. Finally, the LEAP trial only included high-risk infants with a minimal or negative SPT response to peanut and therefore does not address a strategy for those without these risk factors for peanut allergy [7].

\section{How does the LEAP trial affect present guidance for early complementary feeding practices?}

Existing guidelines pertaining to the early introduction of complementary foods have indicated that the introduction of highly allergenic foods, such as peanut, need not be delayed past 4 or 6 months of life. However, they do not actively recommend introduction of peanut between 4 and 6 months of age in high-risk infants, and some of these guidelines specify that certain infants considered at high risk for allergic disease are recommended to first consult an expert [8-14].

The LEAP data provide Level 1 evidence that the practice of early peanut introduction is safe and effective in selected high-risk infants. This study is the first prospective, randomized trial of early peanut intervention and informs provider decision-making regarding high-risk infants, including those already having a positive peanut SPT response but not yet clinically reactive, to receive the benefits noted in the LEAP trial, which might reduce the risk of peanut allergy up to $80 \%$.

Of note, since children with lesser risk factors for peanut allergy were excluded from enrollment in the LEAP trial, there are no prospective, randomized data investigating the benefit or risk of early peanut introduction in the general to low-risk populations. Consequently, this communication's guidance is limited to applying the findings of the LEAP trial to other similar high-risk children in more diverse settings around the world. However, multiple guidelines have not recommended delaying allergen introduction in the general to low-risk populations.

\section{Interim guidance regarding early peanut introduction}

Based on data generated in the LEAP trial and existing guidelines, the following interim guidance is suggested to assist the clinical decision-making of health care providers:

- There is now scientific evidence (Level 1 evidence from a randomized controlled trial) that healthcare providers should recommend introducing peanut-containing products into the diets of "high-risk" infants early on in life (between 4 and 11 months of age) in countries where peanut allergy is prevalent because delaying the introduction of peanut can be associated with an increased risk of peanut allergy.

- Infants with early-onset atopic disease, such as severe eczema, or egg allergy in the first 4 to 6 months of life (see Box 1 for example LEAP criteria), might benefit from evaluation by an allergist or physician trained in management of allergic diseases in this age group to diagnose any food allergy and assist in implementing these suggestions regarding the appropriateness of early peanut introduction. Evaluation of such patients might consist of performing peanut skin testing, in-office observed peanut ingestion, or both, as deemed appropriate after discussion with the family. The clinician can perform an observed peanut challenge for those with evidence of a positive 
peanut skin test response to determine whether they are clinically reactive before initiating at-home peanut introduction. Both such strategies were used in the LEAP trial protocol.

- Adherence in the LEAP trial was excellent (92\%), with infants randomized to consume peanut ingesting a median of $7.7 \mathrm{~g}$ peanut protein (interquartile range: $6.7-8.8 \mathrm{~g}$ ) per week during the first 2 years of the trial compared with a median of $0 \mathrm{~g}$ in the avoidance group (see Box 2 for examples of peanut-containing foods used in the LEAP trial). Although the outcome of the LEAP regimen was excellent, the study does not address use of alternative doses of peanut protein, minimal length of treatment necessary to induce the tolerogenic effect, or potential risks of premature discontinuation or sporadic feeding of peanut.

\section{Box 1 Enrollment Criteria Used in the LEAP Study}

Infants considered at "high risk" as defined by the LEAP study criteria:

Egg allergy: Children with either -

1) A SPT wheal diameter $\geq 6 \mathrm{~mm}$ from exposure to raw hen's egg white and no history of previous egg tolerance,

or

2) A SPT wheal diameter $\geq 3 \mathrm{~mm}$ from exposure to pasteurized hen's egg white and allergic symptoms related to exposure to hen's egg.

Severe eczema: An eczematous rash that -

1) Requires application of topical creams, ointments, or both, containing corticosteroids or calcineurin inhibitors, and that, if the participant is $<6$ months of age, lasted for at least 12 of 30 days on 2 occasions, or, if the participant is $>6$ months of age, lasted for at least 12 of 30 days on two occasions in the last 6 months,

Or

2) Is currently or was previously graded $\geq 40$ using the modified SCORAD evaluation

Example of method of skin prick testing: used in the LEAP study

- SPTs to peanut should be performed in the presence of a negative control and a positive histamine control.

- SPTs should be performed in duplicate, and the maximum wheal diameter of the two SPTs should be calculated and rounded up to the greatest whole millimeter

Of note, in the LEAP trial measurement of IgE to peanut resulted in considerably higher rates of sensitization compared with skin testing, which could lead to numerous unnecessary oral peanut challenges.

\section{Box 2 Examples of Peanut-containing Foods Utilized in the LEAP Trial}

- Smooth peanut butter (1 teaspoon) mixed with milk or with mashed or pureed fruit

- *Bamba ${ }^{\circledR}$ snack (Osem; approximately two thirds of a $1 \mathrm{oz}$. (25 g) bag; 21 sticks of Bamba ${ }^{\oplus}$ ) - for young infants

( $<7$ months), softened with $20-30 \mathrm{ml}$ water or milk and mixed with milk or with mashed or pureed fruit or vegetables

- Peanut soup

- Finely ground peanuts mixed into other foods such as yoghurt *Other foods more customary to particular nations/cultures may be substituted

Whole peanut is not recommended for introduction as this is a choking hazard in children under the age of 4.

\section{Rationale for evaluating and applying this policy to a high-risk population}

The LEAP trial demonstrates that early peanut introduction can be successfully carried out in a high-risk population, such as the population defined in the LEAP trial. However, without intervention by health care providers, there is the potential that such high-risk infants will remain at risk for delayed introduction of solids and allergenic foods into their diet because of the widespread belief that such foods may exacerbate eczema.

There will be more extensive guidelines in the near future from the NIAID Working Group and EAACI Guidelines Group with their multidisciplinary stakeholders. These groups will consider all the available data and determine whether there is sufficient evidence to apply prevention strategies to the general population. However, engagement of the primary care, allergy, and dermatology communities to rapidly implement these findings and change the culture of early feeding practices is essential, and the forthcoming NIAID Working Group's and EAACI Guidelines Group's documents will better clarify a best-practices approach.

\section{Abbreviations}

LEAP: Learning Early about Peanut Allergy; NIAID: National Institute of Allergy and Infectious Diseases; EAACl: European Academy of Allergy and Clinical Immunology; SPT: Skin prick test; ITT: Intention-to-treat; NNT: Number needed to treat.

\section{Competing interests}

The authors declare they have no competing interests associated with this document.

\section{Authors' contribution}

We would like to acknowledge Primary Contributors: David M. Fleischer, MD; Scott Sicherer, MD; Matthew Greenhawt, MD; Dianne Campbell, MB BS FRACP PhD; Edmond S. Chan, MD; Antonella Muraro, MD, PhD, Susanne Halken, MD; Yitzhak Katz, MD; Motohiro Ebisawa, MD, PhD; Lawrence Eichenfield, MD; Hugh Sampson, MD; For the LEAP Trial Team: Gideon Lack, MB, BCh; George Du Toit, MB, BCh; Graham Roberts, DM; Henry Bahnson, $\mathrm{MPH}$; and the Secondary Contributors: Jonathan Hourihane, MD, Jonathan 
Spergel, MD, PhD, Michael Young, MD; Amal Assa'ad, MD; Katrina Allen, BMedSC MB BS FRACP PhD, Susan Prescott, BMedSc MB BS FRACP PhD; Sandeep Kapur, MD; Hirohisa Saito, MD, PhD; loana Agache, MD, Cezmi A. Akdis, MD, PhD, Hasan Arshad, MD, Kirsten Beyer, MD, Anthony Dubois, MD, Philippe Eigenmann, MD, Monserrat Fernandez-Rivas, MD, Kate Grimshaw RD PhD, Karin Hoffmann-Sommergruber, PhD, Arne Host, MD, Susanne Lau MD, Liam O'Mahony, MD, Clare Mills, PhD, Nikolaos Papadopoulos, MD, Carina Venter, BSc, PhD; Nancy Agmon-Levin, MD, Aharon Kessel, MD; Richard Antaya, MD, Beth Drolet, MD; Lanny Rosenwasser, MD Published on behalf of the American Academy of Allergy, Asthma \& Immunology; American Academy of Pediatrics; American College of Allergy, Asthma \& Immunology; Australasian Society of Clinical Immunology and Allergy; Canadian Society of Allergy and Clinical Immunology; European Academy of Allergy and Clinical Immunology; Israel Association of Allergy and Clinical Immunology; Japanese Society for Allergology; Society for Pediatric Dermatology; and World Allergy Organization. Copublished in the Journal of Allergy and Clinical Immunology; the Annals of Allergy, Asthma and Immunology; Allergy, Asthma \& Clinical Immunology; Allergy; and the World Allergy Organization Journal. All authors read and approved the final manuscript.

\section{Acknowledgements \\ FOR THE LEAP TRIAL TEAM \\ Gideon Lack $^{10}$; George Du Toit ${ }^{6}$; Graham Roberts ${ }^{6}$; Henry Bahnson ${ }^{11}$; Mary Feeney ${ }^{10}$ \\ SECONDARY CONTRIBUTORS}

Jonathan Hourihane'; Jonathan Spergel'; Michael Young'; Amal Assa'ad³; Katrina Allen ${ }^{4}$; Susan Prescott ${ }^{4}$; Sandeep Kapur ${ }^{5}$; Hirohisa Saito ${ }^{8}$; Ioana Agache $^{6}$; Cezmi A. Akdis ; Hasan Arshad ${ }^{6}$; Kirsten Beyer ${ }^{6}$; Anthony Dubois ${ }^{6}$; Philippe Eigenmann ${ }^{6}$; Monserrat Fernandez-Rivas ${ }^{6}$; Kate Grimshaw ${ }^{6}$; Karin Hoffmann-Sommergruber ${ }^{6}$; Arne Host ${ }^{6}$; Susanne Lau ${ }^{6}$; Liam O'Mahony ${ }^{6}$; Clare Mills $^{6}$; Nikolaos Papadopoulos ${ }^{6}$; Carina Venter ${ }^{6}$; Nancy Agmon-Levin ${ }^{7}$; Aharon Kessel $^{7}$; Richard Antaya ${ }^{9}$; Beth Drolet ${ }^{9}$; Lanny Rosenwasser ${ }^{10}$

${ }^{1}$ American Academy of Allergy, Asthma \& Immunology (AAAAl), Milwaukee, WI, United States of America ${ }^{2}$ American Academy of Pediatrics (AAP), Chicago, IL, United States of America

${ }^{3}$ American College of Allergy, Asthma \& Immunology (ACAAI), Chicago, IL, United States of America

${ }^{4}$ Australasian Society of Clinical Immunology and Allergy (ASCIA), Brookvale, NSW, Australia

${ }^{5}$ Canadian Society of Allergy and Clinical Immunology (CSACI), Orleans, ON, Canada

${ }^{6}$ European Academy of Allergy and Clinical Immunology (EAACl), Zurich, Switzerland

${ }^{7}$ Israel Association of Allergy and Clinical Immunology (IAACI), Tel-Hashomer, Israel

${ }^{8}$ Japanese Society for Allergology (JSA), Tokyo, Japan

${ }^{9}$ Society for Pediatric Dermatology (SPD), Indianapolis, IN, United States of

America

${ }^{10}$ World Allergy Organization (WAO), Milwaukee, WI, United States of America

${ }^{11}$ Rho Federal Systems Division Inc., Chapel Hill, NC, United States of America

\begin{abstract}
Author details
${ }^{1}$ American Academy of Asthma, Allergy \& Immunology (AAAAI), Milwaukee, WI, USA. ${ }^{2}$ American Academy of Pediatrics (AAP), Chicago, IL, USA. ${ }^{3}$ American College of Allergy, Asthma, and Immunology (ACAAI), Chicago, IL, USA. ${ }^{4}$ Australasian Society of Clinical Immunology and Allergy (ASCIA), Brookvale, Australia. ${ }^{5}$ Canadian Society of Allergy and Clinical Immunology (CSACI), Orleans, ON, Canada. ${ }^{6}$ European Academy of Allergy and Clinical Immunology (EAACI), Zurich, Switzerland. ${ }^{~}$ Israel Association of Allergy and Clinical Immunology (IAACI), Tel-Hashomer, Israel. ${ }^{8}$ Japanese Society for Allergology (JSA), Tokyo, Japan. ${ }^{9}$ Society for Pediatric Dermatology (SPD), Indianapolis, IN, USA. ${ }^{10}$ World Allergy Organization (WAO), Milwaukee, WI, USA.
\end{abstract}

Received: 9 June 2015 Accepted: 10 June 2015

Published online: 03 August 2015

\section{References}

1. Nwaru BI, Hickstein L, Panesar SS, Muraro A, Wergel T, Cardona V, et al. The epidemiology of food allergy in Europe: a systematic review and metaanalysis. Allergy. 2014;69:62-75.
2. Osborne NJ, Koplin JJ, Martin PE, Gurrin LC, Lowe AJ, Matheson MC, et al. Prevalence of challenge-proven IgE-mediated food allergy using population-based sampling and predetermined challenge criteria in infants. J Allergy Clin Immunol. 2011;127:668-76.

3. Venter C, Hasan Arshad S, Grundy J, Pereira B, Bernie Clayton C, Voigt K, et al. Time trends in the prevalence of peanut allergy: three cohorts of children from the same geographical location in the UK. Allergy. 2010;65:103-8.

4. Sicherer SH, Muñoz-Furlong A, Godbold JH, Sampson HA. US prevalence of self-reported peanut, tree nut, and sesame allergy: 11-year follow-up. J Allergy Clin Immunol. 2010;125:1322-6.

5. Soller L, Ben-Shoshan M, Harrington DW, Fragapane J, Joseph L, St Pierre Y, et al. Overall prevalence of self-reported food allergy in Canada. J Allergy Clin Immunol. 2012;130:986-8.

6. Amoah AS, Obeng BB, Larbi IA, Versteeg SA, Aryeetey Y, Akkerdaas JH, et al. Peanut-specific lgE antibodies in asymptomatic Ghanaian children possibly caused by carbohydrate determinant cross-reactivity. J Allergy Clin Immunol. 2013;132:639-47.

7. DuToit G, Roberts G, Sayre PH, Bahnson HT, Radulovic S, Santos AF, et al. Randomized trial of peanut consumption in infants at risk for peanut allergy. N Engl J Med. 2015;372:803-13.

8. Greer FR, Sicherer SH, Burks AW. Effects of early nutritional interventions on the development of atopic disease in infants and children: the role of maternal dietary restriction, breastfeeding, timing of introduction of complementary foods, and hydrolyzed formulas. Pediatrics. 2008;121:183-91.

9. Muraro A, Halken S, Arshad SH, Beyer K, Dubois AE, Du Toit G, et al. EAACl food allergy and anaphylaxis guidelines. Primary prevention of food allergy. Allergy. 2014;69:590-601.

10. de Silva D, Geromi M, Halken S, Host A, Panesar SS, Muraro A, et al. Primary prevention of food allergy in children and adults: systematic review. Allergy. 2014;69:581-9.

11. Fleischer DM, Spergel JM, Assa'ad AH, Pongracic JA. Primary prevention of allergic diseases through nutritional interventions. J Allergy Clin immunol Pract. 2013;1:29-36.

12. Chan ES, Cummings C. Canadian Paediatric Society, Community Paediatrics Committee and Allergy Section. Dietary exposures and allergy prevention in high-risk infants: A joint statement with the Canadian Society of Allergy and Clinical Immunology. Paediatr Child Health. 2013;18:545-54.

13. Agostoni C, Decsi T, Fewtrell M, Goulet O, Kolacek S, Koletzko B, et al. Complementary feeding: a commentary by the ESPGHAN committee on nutrition. J Pediatr Gastroenterol Nutr. 2008:46:99-110.

14. Australasian Society of Clinical Immunology and Allergy (ASCIA). ASCIA infant feeding advice: Available at: http://www.allergy.org.au/images/ stories/aer/infobulletins/2010pdf/ASCIA_Infant_Feeding_Advice_ 2010.pdf. (Accessed April 2, 2015)

\section{Submit your next manuscript to BioMed Central and take full advantage of:}

- Convenient online submission

- Thorough peer review

- No space constraints or color figure charges

- Immediate publication on acceptance

- Inclusion in PubMed, CAS, Scopus and Google Scholar

- Research which is freely available for redistribution 